\title{
La formación investigativa en los estudiantes de licenciatura en Educación Básica. Caso Universidad Técnica del Norte, Ecuador
}

The research training in the students of Basic Education degree. Case of the Technical University of the North, Ecuador

Formação em investigação para estudantes universitários do Ensino Básico. Caso da Universidade Técnica do Norte, Equador

\author{
FRANK EDISON GUERRA REYES ${ }^{1}$ \\ MIGUEL EDMUNDO NARANJO TORO²
}

\begin{abstract}
RESUMEN
La enseñanza-aprendizaje de los procesos investigativos en las instituciones de educación superior reproduce prácticas didácticas sustentadas en la retención-repetición de teoría y metodología científica. Esta realidad requiere de innovación permanente para superar las limitaciones del tradicionalismo didáctico. El propósito de este estudio fue fortalecer los procesos de investigación formativa con los estudiantes de la carrera de Licenciatura en Educación Básica de la Universidad Técnica del Norte - Ecuador. Se empleó investigación documental e investigación-acción enmarcadas en los planteamientos de la Enseñanza para la Comprensión. Como técnicas se utilizó: revisión documental, entrevistas a docentes y estudiantes, análisis de contenido y triangulación. Al final, se encontró que la investigación formativa constituye un área primordial para la formación de los futuros docentes, pero aún se tienen ciertas falencias en su comprensión y aplicación. Como estrategias para la acción se determinaron: aprendizaje basado en problemas, monografías investigativas, club de revistas científicas y seminarios investigativos.
\end{abstract}

Palabras clave: investigación formativa; formación docente; educación básica; investigación-acción; enseñanza para la comprensión.

\begin{abstract}
The teaching-learning of research processes in higher education institutions reproduces didactic practices based on the retention-repetition of theory and scientific methodology. This reality requires permanent innovation to overcome the limitations of didactic traditionalism. The purpose of this study was to strengthen the processes of formative research with the students of the Bachelor's degree in Basic Education at the Technical University of the North - Ecuador. Documentary research was used and research-action framed in the
\end{abstract}


approaches of the Teaching for Understanding. The techniques used were: documentary review, teacher and student interviews, content analysis and triangulation. At the end, it was found that formative research constitutes a primordial area for the formation of future teachers, but there are still certain deficiencies in its understanding and application. As strategies for action were determined: problem-based learning, research monographs, scientific journal club and research seminars.

Keywords: formative research; teacher training; basic education; action research; teaching for understanding.

\section{RESUMO}

O ensino-aprendizagem dos processos de investigação nas instituições de ensino superior reproduz práticas didácticas baseadas na retenção-repetição da teoria e metodologia científica. Esta realidade exige inovação permanente para superar as limitações do tradicionalismo didáctico. O objectivo deste estudo era reforçar os processos de investigação formativa com os alunos do Bacharelato em Educação Básica da Universidade Técnica do Norte - Equador. Foi utilizada investigação documental e investigação-acção no âmbito das abordagens do Ensinamento para a Compreensão. As técnicas utilizadas foram: revisão documental, entrevistas a professores e estudantes, análise de conteúdos e triangulação. No final, verificou-se que a investigação formativa constitui uma área primária para a formação de futuros professores, mas ainda existem certas deficiências na sua compreensão e aplicação. Foram identificadas estratégias de acção: aprendizagem baseada em problemas, monografias de investigação, clube de revistas científicas, e seminários de investigação.

Palavras-chave: Investigação em formação; formação de professores; educação básica; investigação de acção; ensino para a compreensão.

\section{INTRODUCCIÓN}

Al iniciar la tercera década del siglo XXI, es común escuchar que nuestros jóvenes son diferentes a las generaciones pasadas y que, por lo tanto, aprenden de manera diversa. En contraposición, la enseñanza que reciben continua casi inmutable. De manera específica, la realidad cotidiana de la enseñanza-aprendizaje de los procesos investigativos continúa reproduciendo prácticas didácticas tradicionalistas, sustentadas únicamente en la retención-repetición de teoría y metodología científica consignada en libros académicos.

Esta realidad requiere de constante innovación si se quiere superar las limitaciones en estrategias didácticas actualizadas y fundamentadas coherentemente en la ciencia pedagógica y metodología investigativa postmoderna. Al respecto Aldana de Becerra (2012), manifiesta que:

La investigación viene adquiriendo cada vez mayor importancia en la educación superior por varias razones, entre ellas, la tendencia a mejorar la calidad de la educación, la necesidad de producir, difundir y apropiar conocimiento de manera adecuada y competitiva, la necesidad de dar respuesta a los múltiples problemas sociales, desde una perspectiva cientí- 
fica y humanista y por la intención de formar profesionales capaces de generar conocimiento o al menos ser sensibles a la investigación [...] (p. 369).

A partir del análisis de la situación problemática, se arribó a la contradicción evidenciada entre la necesidad del empleo de la investigación formativa y el bajo nivel de su aplicación en el proceso de enseñanza-aprendizaje universitario, por lo que se definió el siguiente problema de investigación: ¿Cómo fortalecer los procesos de investigación formativa universitaria con los estudiantes de la carrera de Licenciatura en Educación Básica en la Universidad Técnica del Norte, Ecuador?

La investigación realizada se inscribe en el paradigma constructivista, con énfasis en la investigación-acción. Debido al vínculo establecido entre la investigación y el desarrollo didáctico en la formación universitaria, el constructivismo facilita un sendero académico para orientar el proceso de enseñanza-aprendizaje, coincidente con la política nacional y sus normativas en el contexto ecuatoriano.

Al ser uno de los paradigmas vigentes y contemporáneos utilizados en la formación profesional, se han seguido diferentes perspectivas constructivistas; entre ellas, el enfoque propuesto por Jean Piaget, el constructivismo social de César Coll y la Enseñanza para la Comprensión de David Perkins y colaboradores. Según Serulnikov y Suárez (2010), Piaget, considerado como el gestor del constructivismo contemporáneo, asume una relación dinámica para la construcción del conocimiento. El sujeto interpreta el mundo en que se desarrolla. Los aprendizajes se reestructuran (asimilan-acomodan) como producto de la interacción de lo que conoce previamente con lo nuevo, en concordancia con sus necesidades personales y su desarrollo evolutivo. Para Coll (1997), el aprendizaje no es una construcción individual, sino más bien una construcción social para formar personas justas, innovadoras y solidarias. Es, en la vida en sociedad, como los antepasados median con la cultura que se transmite de generación en generación a través de distintas interacciones entre las personas. Boix Mansilla y Gardner (apud Stone Wiske, 2008), sostienen que el proceso de enseñanza-aprendizaje posibilita el desarrollo humano a través de la transmisión del acervo cultural en distintos ámbitos de interacción comunicativas que las personas despliegan a lo largo de su vida, no solamente en las aulas formativas sino en todo contexto sociocultural.

Como resultado del desarrollo teórico del constructivismo, surgió la Enseñanza para la Comprensión (EpC) en su interpretación pedagógica. Según Perkins (2008, p. 21), “... el aprendizaje es una consecuencia del pensamiento. Solo es posible retener, comprender y usar activamente el conocimiento mediante experiencias de aprendizaje en las que los alumnos reflexionan sobre lo que están aprendiendo y con lo que están aprendiendo". La Enseñanza para la Comprensión, llamada "constructivismo del desempeño" (Perrone, apud Stone Wiske, 2008), es un enfoque cimentado en las bases del constructivismo que procura ayudar a los docentes en la creación de una nueva pedagogía. Pretende apoyar a los docentes facilitando un marco de referencia que explica, por un lado, cómo se construyen comprensiones profundas; $y$, por otro, destaca la importancia que tienen las comprensiones en el desarrollo de un pensamiento cada vez más complejo que permita a los estudiantes resolver problemas de manera flexible y crear productos nuevos y significativos para su cultura. Toma en cuenta los conocimientos previos, al centrar su acción sobre las concepciones alternativas que los estudiantes poseen de su entorno y la manera en que este funciona, así como de la responsabilidad que tienen en la construcción de su propio aprendizaje. 
Con respecto a la necesaria interacción investigativo-didáctico-curricular, Lawrence Stenhouse (2007) expresa:

Cuando ustedes realizan una investigación en la acción, lo crucial es una especificación estricta, saber qué es lo que están haciendo. Y saber que lo que están haciendo significa redactar un curriculum. Significa tener absolutamente claro cuál es el curriculum con el que están experimentando. Piensen en los hermanos WRIGHT y en el aeropuerto. Existía un diseño, unos planos o dibujos. Para hacer la investigación fue preciso construir el aeroplano. De nada sirve que corran por la pista agitando los brazos. Han de crear realmente alguna especie de instrumento. Y si están enseñando con un espíritu de indagación, el curriculum constituye la especificación de la investigación en la acción... La investigación en la acción se relaciona con el curriculum de tal modo que éste constituye una definición y una especificación del procedimiento experimental (p. 91).

En síntesis, se desarrolló un estudio que tributa a los planteamientos de una didáctica comprensivo-investigativa (Basagoiti, Bru-Martin, Lorenzana, 2001; Elliot, 1990 y 2005; Kemmis y McTaggart, 1998; Latorre, 2005; McKernan, 1999; y, Stenhouse, 2007).

\section{METODOLOGÍA}

Se empleó investigación documental e investigación-acción, enmarcadas en los planteamientos de la Enseñanza para la Comprensión. Como técnicas se utilizó: revisión documental, entrevistas a docentes y estudiantes, análisis de contenido y triangulación. En la figura 1, se presenta, de manera resumida, la metodología empleada.

\section{Investigación-acción}

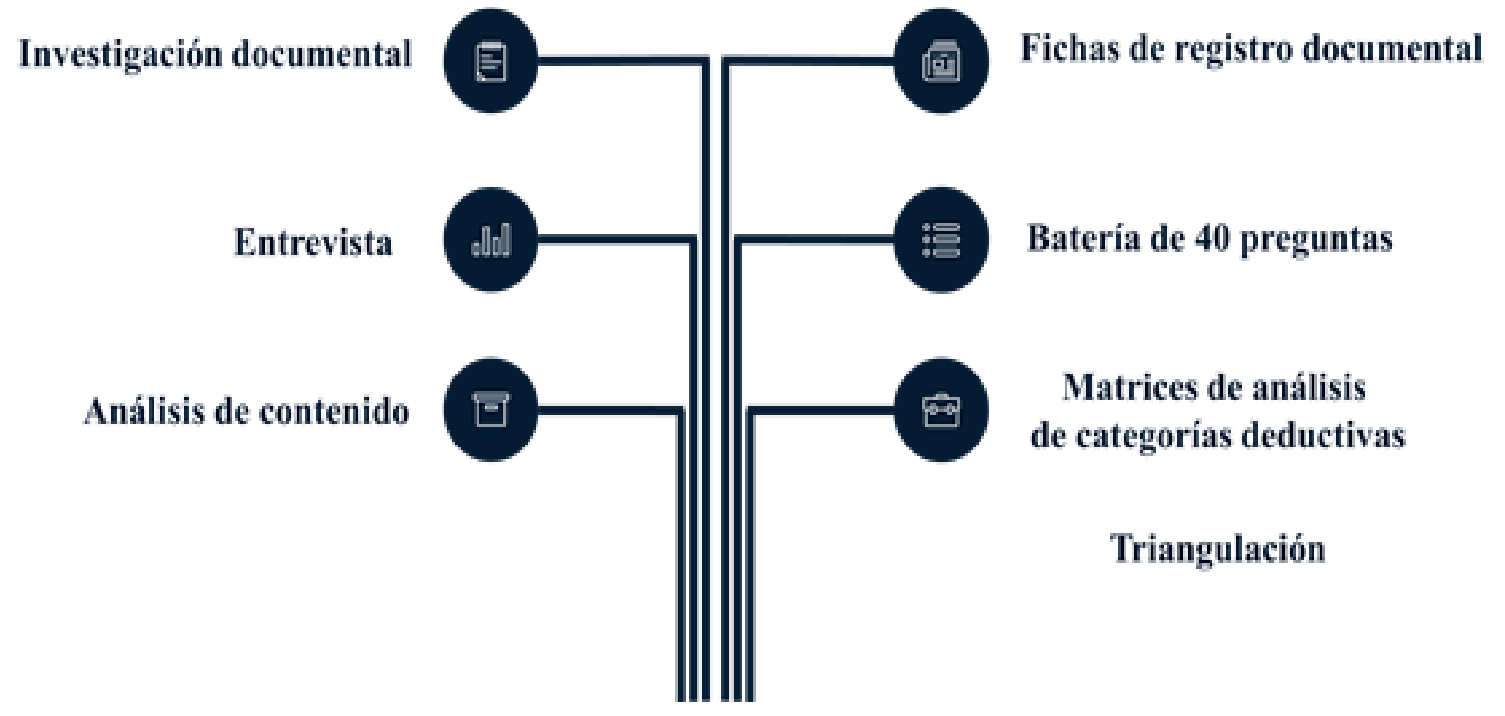

Figura 1. Metodología empleada en la investigación 


\section{Población}

Docentes y estudiantes de la Carrera de Licenciatura en Educación Básica de la Universidad Técnica del Norte, de Ecuador. La población docente, al momento de la investigación, fue de 14, mientras que la de estudiantes fue de 154.

\section{Muestra}

El criterio de selección fue de informantes clave, integrado por 14 estudiantes auxiliares de investigación (tres del $2^{\circ}$ nivel, dos del $3^{\circ}$, cinco del $4^{\circ}$ y 4 del $5^{\circ}$ nivel de estudios o semestre de formación profesional docente). Al ser una carrera de reciente creación, solamente consta de cinco niveles formativos de un total de ocho. Como criterio de inclusión se integró a los docentes y otros estudiantes de la carrera de Licenciatura en educación básica (EB), quiénes aportaron por voluntariedad, a la investigación. Se excluyeron a los docentes y estudiantes que no demostraron interés en participar. Tampoco se consideró a los estudiantes de primer semestre, ya que, al iniciar la carrera, todavía no cuentan con el conocimiento y la experiencia suficiente del proceso enseñanza-aprendizaje implementado en la carrera de EB. Al final, se tuvo la participación de 14 estudiantes auxiliares de investigación, 11 docentes y 65 estudiantes de distintos niveles de formación en la Licenciatura en Educación Básica (18 de segundo, 14 de tercero, 14 de cuarto y 19 de quinto).

\section{Las técnicas e instrumentos}

\section{a. Entrevista}

De acuerdo con Moriña (2017), “...cuando se realiza una entrevista es importante que se estimulen positivamente las ganas de hablar de quien narra, destacando las aportaciones científicas de su contribución, haciéndole sentir la importancia de su testimonio para el proyecto de investigación" (p. 51). Para lograrlo, sugiere el diseño de un guion de entrevista o un banco de preguntas.

A continuación, en la tabla 1, se detalla el proceso de determinación de las Categorías deductivas que orientaron el proceso de recolección y análisis de datos. Al final, con su empleo, se construyó una guía de entrevista conformada como un banco de 40 preguntas, que fue aplicado a docentes y estudiantes, con variaciones mínimas en la redacción, en consonancia con la función didáctica. 
Tabla 1.

Categorías deductivas que orientaron el proceso de recolección de datos

Objetivo específico
Conocer la situación inicial
del empleo de la investigación
para la enseñanza-aprendizaje
de los contenidos de estudio en
la Licenciatura en Educación
Básica de la Universidad Téc-
nica del Norte (UTN)

Interpretar los hallazgos rela-
cionados con el empleo de la
investigación para la enseñan-
za-aprendizaje de los conteni-
dos de estudio en la Licencia-
tura en Educación Básica de la
Universidad Técnica del Norte
(UTN)

Profundizar teóricamente en la integración de la investigación en el proceso de enseñanza-aprendizaje en la Licenciatura en Educación Básica de la Universidad Técnica del Norte.

Implementar acciones de integración de la investigación en el proceso de enseñanza-aprendizaje en la Licenciatura en Educación Básica de la Universidad Técnica del Norte

Evaluar las acciones desarrolladas para la integración de la investigación en el proceso de enseñanza-aprendizaje en la Licenciatura en Educación Básica de la Universidad Técnica del Norte.

\section{Categoría o concepto central}

Situación inicial del empleo de la investigación en el Proceso enseñanza-aprendizaje (PEA).

Hallazgos del empleo de la investigación en el PEA.

Teoría sobre integración sobre empleo de investigación en el PEA.
Consiste en la búsqueda y construcción teórica sobre la integración de la investigación en el PEA.

Definición

El empleo de la investigación en el PEA consiste en un enfoque didáctico que permite al docente desarrollar en los educandos competencias que les permitan realizar una investigación en su contexto

Los hallazgos de la investigación en el PEA consisten en el análisis e interpretación de los datos relacionados con el enfoque didáctico implementado.

Consiste en las acciones y propuestas posibles para la integración de la investigación en el PEA.

\section{Indicadores}

- $\quad$ Finalidad (memorizar disciplinas, desarrollar competencias y/o participación social)

- Elementos didácticos (objetivos, contenidos, métodos, medios y evaluación)

- Contexto (problemas comunidad y proyección social)

- Docentes (saber profesional e implementación didáctica)

- $\quad$ Estudiantes (percepciones, potencialidades y vinculación)

- Reportes de estatus de investigación en disciplinas.

- Análisis de metodología $\mathrm{y}$ argumentos presentados en artículos y libros.

- Construcción de estado del arte y marco teórico.

- Ensayos teóricos con esquemas de investigación.

- El club de las revistas.

- Seminarios investigativos.

- Aprendizaje Basado en Problemas.

tegración de la investigación en el PEA.
- $\quad$ Ejercicios de diseño de anteproyectos de investigación.

- Vinculación de estudiantes a proyectos de investigación.

- $\quad$ Elaboración de una monografía investigativa.

Fuente: Blessinger. y Carfora, 2014; Díaz-Barriga y Hernández-Rojas, 2010; Hernández-Sampieri y Mendoza-Torres, 2018; Kemmis, McTaggart y Nixon, 2013; Mancovsky y Moreno, 2015; Parra-Moreno, 2004; Schettini y Cortazzo, 2015; y, Torres (s.f.). 


\section{Registro documental}

De acuerdo con Escobar (2020), existen instrumentos diseñados por universidades que posibilitan sistematizar manualmente la información obtenida de documentos impresos y electrónicos, conocidos como resúmenes analíticos especializados o Fichas RAE. Estos instrumentos ponen en evidencia una estructura referencial, con información prioritaria de los conceptos y categorías más importantes, así como de los aportes metodológicos y las conclusiones a las que llegaron los investigadores o autores de las publicaciones científicas. En la tabla 2, se muestra la ficha utilizada.

Tabla 2.

Ficha de registro documental $R A E$

Título
Autor(a)/es
Tipo de documento
Lugar (ciudad, país de origen)
Tema
Objetivos
Categorías
Método de investigación
Síntesis del documento (Resumen)
Resultados
Comentarios y reflexiones
Referencias del documento

Fuente: (Escobar, 2020)

\section{Análisis de contenido}

El Análisis de Contenido constituye una de tantas técnicas para realizar una investigación cualitativa. Autores como Carrero, Soriano y Trinidad (2012), la referencian para la generación de contenidos en su teoría fundamentada. Por su parte Gibbs (2012), la menciona para el análisis de datos cualitativos.

El Análisis de Contenido ofreció la posibilidad para investigar sobre la naturaleza del discurso. A través de este procedimiento se analizó y extrajo las categorías emergentes determinadas en las entrevistas con docentes y estudiantes. Según Bardin (1996), la finalidad analítica primordial que resuelven las técnicas de "Análisis de Contenido" es la identificación y explicación de las representaciones cognoscitivas que otorgan el sentido a todo relato comunicativo (p.5).

Luego de la transcripción de las entrevistas, se realizó el análisis de su contenido, teniendo en cuenta la variable, dimensiones e indicadores asumidos en la investigación, los que se resumen en la Figura 2. 


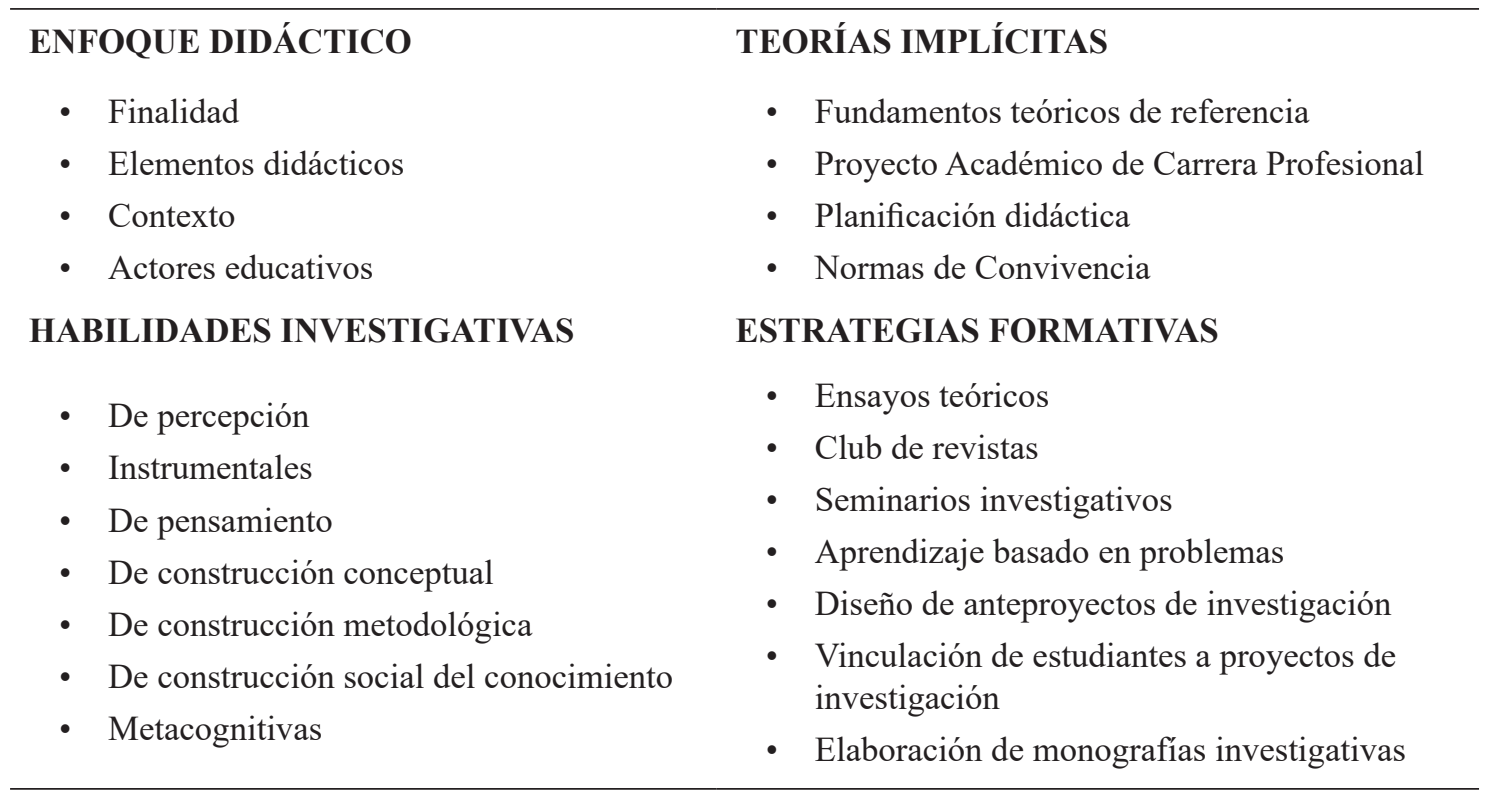

Figura 2. Bosquejo esquemático para análisis de contenido

\section{RESULTADOS Y DISCUSIÓN}

Los resultados se desagregan en tres componentes, a saber: entrevista a estudiantes, entrevista a docentes e investigación documental.

\subsection{Entrevista a estudiantes}

Finalidad: a pesar de la percepción inicial de que el proceso didáctico se enmarca en el desarrollo de habilidades y participación en la sociedad, prevalecen sus vivencias de una enseñanza-aprendizaje memorística en las disciplinas que estudian.

Elementos didácticos: determinan que sus docentes preparan las clases de manera frecuente, con énfasis en los contenidos, la metodología y los recursos didácticos.

Contexto: limitada integración de problemáticas contextuales en las clases recibidas.

Saber profesional e implementación didáctica: según su discernimiento, sus docentes casi no enfatizan en la investigación formativa; consideran que ellos, están preparados, de manera básica, para la ejecución de investigaciones.

Habilidades investigativas: han desarrollado sensibilidad ante los fenómenos educativos, con un nivel aceptable en las habilidades para leer, escribir, escuchar y hablar; la formación recibida les habilita para pensar de manera crítica y autónoma, de hecho, consideran que pueden generar, organizar y defender sus ideas; por otra parte, manifiestan inseguridad para diseñar procedimientos e instrumentos investigativos; el aprendizaje de habilidades para la construcción del conocimiento, está en proceso; y, por último, manifiestan limitaciones para desarrollar una investigación de manera autónoma. 
Percepciones, potencialidades y vinculación: perciben la investigación como una tarea complicada, impuesta e incómoda; no obstante, también manifiestan que les ha apoyado para la recolección de información y la lectura de textos académicos.

Teorías implícitas. Fundamentos teóricos de referencia: en general, no han interiorizado ningún referente teórico en particular. Entre los ejemplos, refieren nombres de filósofos de la antigüedad, novelistas y pocos nombres de psicopedagogos contemporáneos.

Teorías implícitas. Proyectos educativos institucionales: refieren ejemplos concretos sobre la filosofía, principios y valores de la Universidad.

Teorías implícitas. Proyecto académico de carrera profesional: no evidencian participación en el diseño de los proyectos educativos de la Carrera de Educación Básica.

Teorías implícitas. Normas de convivencia: no hay acuerdo con relación a si el contexto ha condicionado su práctica pre profesional. Como valores, establecen: respeto, responsabilidad y honestidad intelectual.

Estrategias formativas. Ensayos teóricos: limitada referencia a su empleo, por lo cual, manifiestan que no se encuentran preparados para su elaboración.

Estrategias formativas. Club de revistas: aducen haber participado en la elaboración de artículos científicos, a los que califican con un nivel alto de dificultad. Sin embargo, como parte del "plan de acción" bosquejado en el proyecto: "Apoyo a la formación investigativa en los estudiantes de Licenciatura en Educación Básica", 14 estudiantes auxiliares de investigación participaron en la recolección de información para este estudio, en cuatro grupos colaborativos. Por otra parte, están construyendo artículos científicos y de revisión que se publicarán en una revista monográfica de la Facultad de Educación, Ciencia y Tecnología de la UTN.

Estrategias formativas. Seminarios investigativos: no han participado en estos eventos.

Estrategias formativas. Aprendizaje basado en problemas (ABP): manifestaron haber trabajado en pocas asignaturas, a saber: Didáctica, Psicología y Diseño Curricular

Estrategias formativas. Diseño de anteproyectos de investigación: la mayoría manifiesta no haber participado en el diseño de anteproyectos de investigación.

Estrategias formativas. Vinculación de estudiantes a proyectos de investigación: expresan que son pocos los maestros que los vinculan en proyectos de investigación.

Estrategias formativas. Elaboración de monografías investigativas: consideran factible y necesaria, la elaboración de monografías investigativas como estrategia en su formación docente-investigativa. 


\subsection{Entrevista a docentes}

Finalidad: apego a un proceso didáctico relacionado con el desarrollo de habilidades.

Elementos didácticos: la metodología constituye el componente esencial del proceso enseñanza-aprendizaje (PEA) que implementan a través de: diseño de proyectos, estudio de casos, ABP; como recursos didácticos enlistan: libros físicos (en papel) y artículos científicos.

Contexto: revelan ejemplos disciplinares más que de respuesta al contexto social.

Docentes. Saber profesional e implementación didáctica: según ellos, el empleo de la investigación formativa se gesta en las disciplinas que enseñan. De manera prioritaria en el trabajo en las Cátedras integradoras que se implementan en cada nivel formativo.

Docentes. Habilidades investigativas: manifiestan sensibilidad para integrar en sus clases (inclusión educativa, rescate de tradiciones y valores culturales, igualdad de derechos y desarrollo de habilidades tecnológicas); manifiestan que apoyan a los estudiantes a pensar de manera crítica y autónoma, a través de la organización de debates, foros y plenarias; además, que los preparan para generar, organizar y defender sus ideas; en cuanto a la adquisición de las competencias para el diseño de procedimientos e instrumentos para buscar, recuperar y/o generar información, reconocen que todavía es una tarea pendiente; por otra parte, no ejemplifican experiencias concretas de procesos de construcción del conocimiento; por último, sugieren algunas limitaciones al momento de desarrollar una investigación de manera autónoma.

Estudiantes. Percepciones, potencialidades y vinculación: a su juicio, los estudiantes estarían preparados, de manera elemental, para la ejecución de investigaciones, con énfasis en la recolección de información y lectura de textos.

Teorías implícitas. Fundamentos teóricos de referencia: expresan que han integrado la investigación cualitativa en su trabajo docente, el cual se apoya en el empleo de bases de datos académicas y en menor proporción, con el uso de libros físicos.

Teorías implícitas. Proyectos educativos institucionales: reconocen la filosofía, principios y valores institucionales; por otro lado, pocos evidencian participación en los proyectos educativos de la Carrera de Educación Básica.

Teorías implícitas. Proyecto académico de carrera profesional: manifiestan haber integrado la investigación formativa en el PEA.

Teorías implícitas. Normas de convivencia: limitada evidencia ejemplificada de la influencia del contexto en su quehacer docente. Como valores, establecen: respeto y tolerancia.

Estrategias formativas. Ensayos teóricos: no suelen elaborar ensayos teóricos.

Estrategias formativas. Club de revistas: casi todos manifiestan y comunican ejemplos de artículos científicos desarrollados; como limitación expresan, problemas para adecuarse a normativas y formatos de las revistas científicas; $y$, como fortaleza, su habilidad para la búsqueda y organización de la información. 
Estrategias formativas. Seminarios investigativos: han participado en eventos organizados en la Universidad Técnica del Norte (UTN), pero también a nivel nacional e internacional; estos escenarios les han generado opciones de capacitación en habilidades investigativas.

Estrategias formativas. Aprendizaje basado en problemas (ABP): determinan un alto nivel de importancia y uso frecuente en sus interacciones didácticas.

Estrategias formativas. Diseño de anteproyectos de investigación: pocos han participado con sus estudiantes en la elaboración de anteproyectos de investigación.

Estrategias formativas. Vinculación de estudiantes a proyectos de investigación: califican un nivel básico de vinculación estudiantil en el desarrollo de proyectos de investigación, debido a la limitada asignación laboral, pero también a una escasa predisposición estudiantil para involucrarse en las tareas investigativas.

Estrategias formativas. Elaboración de monografías investigativas: consideran practicable su implementación; sin embargo, requieren preparar, de manera sistemática, a los estudiantes en el dominio de las normas APA y en el manejo de metodología científica.

\subsection{Investigación documental}

Las problemáticas encontradas en la muestra documental determinan un escaso uso de la investigación formativa en la educación superior. Se han publicado hallazgos que dan cuenta de la insuficiente motivación de los estudiantes para integrarse en procesos, programas o proyectos investigativos, y también, en el inadecuado uso de la investigación formativa por parte de los docentes, como modelo didáctico. Según Wiemer (2019), la implementación del aprendizaje basado en la indagación sigue obstaculizada por las prácticas tradicionalistas de interaprendizaje, prevalentes en las instituciones de educación superior.

Por otra parte, se reconoce a la investigación formativa como eje fundamental para la formación de los futuros profesionales de la docencia, con habilidades relacionadas con el pensamiento crítico, con capacidad para el aprendizaje permanente, para la búsqueda de problemas irresueltos y como estrategia didáctica para el planteamiento de soluciones a las problemáticas de su contexto social, así como las que se presenten en sus tareas cotidianas. Según Ludwig Huber, 2009, (apud Mieg, 2019, p. 15, traducido):

A diferencia de otros métodos de aprendizaje, el aprendizaje basado en la indagación se caracteriza por el hecho de que los alumnos configuran, aprenden y deliberan sobre el proceso de un proyecto de investigación, que tiene como objetivo obtener ideas que sean de interés para terceros, haciéndolo durante todo el proceso. Desde desarrollar preguntas e hipótesis seleccionar e implementar los métodos, hasta probar y presentar los resultados, ya sea trabajando de forma independiente o en colaboración activa con un proyecto global.

Por último, la investigación formativa es caracterizada como modelo didáctico que permite acceder a los contenidos y formas culturales, así como para profundizar en la construcción del conocimiento. 


\section{CONCLUSIONES}

La triangulación de los resultados, permitió establecer, las siguientes conclusiones:

Enfoque didáctico: a pesar de la persistencia del modelo tradicionalista que prioriza a los contenidos, métodos y recursos, como elementos didácticos, comienzan a desarrollarse y validarse, a través de la investigación formativa, procesos que promueven el desarrollo integral, el trabajo colaborativo, aprendizaje basado en problemas y las propuestas situadas como respuesta a las demandas sociales.

Habilidades investigativas: surgimiento de un PEA sensible con la inclusión, tradiciones, valores, derechos y las TIC. Igualmente, valoración de objetivos y desarrollos didácticos que promueven la formación profesional crítica e investigativa, a pesar de las limitaciones y obstáculos cognitivos, procedimentales y actitudinales para el desarrollo de estas habilidades investigativas, de parte de los actores educativos.

Teorías implícitas: planificación docente exhaustiva, aunque inadecuada en torno a la aplicación de un PEA sustentado en la investigación. No obstante, comienzan a implementarse prácticas didácticas orientadas a la investigación cualitativa e investigación-acción, en el desarrollo de los "Proyectos Integradores de Saberes" (PIS), desarrollados en cada nivel formativo como parte de las Cátedras Integradoras. Por último, apreciación actitudinal por parte de docentes y estudiantes, con la prevalencia de valores como: respeto, responsabilidad y honestidad, como normas básicas de conducta académica.

Estrategias formativas: debido a la baja motivación estudiantil y el escaso tiempo determinado para que los docentes investiguen, se gestó una limitada incorporación estudiantil a proyectos desarrollados por docentes; no obstante, esta realidad, motivó el diseño e implementación del proyecto: "Apoyo a la formación investigativa en los estudiantes de Licenciatura en Educación Básica". Proyecto que se ha constituido en el "plan de acción" para que los docentes-investigadores y los 14 estudiantes auxiliares de investigación, constituyan el Grupo de Investigación "luz-a-zul". Como producto, los estudiantes elaboraron cuatro artículos científicos que serán editados y publicados en un monográfico con el sello editorial UTN. Asimismo, se desarrollará un Congreso sobre investigación formativa con la invitación a docentes de varias universidades del país.

\section{REFERENCIAS}

Aldana de Becerra, G. (2012). La formación investigativa: su pertinencia en pregrado. Revista Virtual Universidad Católica del Norte, (35), 367-379. https://revistavirtual. ucn.edu.co/index.php/RevistaUCN/article/view/366

Bardin, L. (1996). Análisis de contenido (2a Ed.). Akal.

Basagoiti, M., Bru-Martin, P. y Lorenzana, C. (2001). Investigación-accion participativa. Acsur.

Blessinger, P. y Carfora, J. (Ed.). (2014). Inquiry-based learning for the arts, humanities and social sciences. A conceptual and practical resources for educators. Emerald Publishing Limited. 
Carrero, V., Soriano, R., y Trinidad, A. (2012). Teoría fundamentada. El desarrollo de teoría desde la generalización conceptual. Centro de Investigaciones Sociológicas.

Coll, C. (1997). Aprendizaje escolar y construcción del conocimiento. Paidós.

Díaz-Barriga, F. (2010). Estrategias docentes para un aprendizaje significativo. Una interpretación constructivista. (2a . Ed.). McGrawHill.

Elliot, J. (1990). La investigación-acción en educación. Morata.

Elliot, J. (2005). El cambio educativo desde la investigación-acción (4ª . Ed.). Morata.

Escobar, G. (2020). Metodología cualitativa para educación y ciencias sociales I. (Curso-taller). RedDOLAC.

Gibbs, G. (2012). El análisis de datos cualitativos en investigación cualitativa. Morata.

Hernández-Sampieri, R. y Mendoza-Torres, Ch. P. (2018). Metodología de la investigación. Las rutas cuantitativa, cualitativa y mixta. McGrawHill Education

Kemmis, S. y McTaggart, R. (1998). Cómo planificar la investigación-acción. Laertes.

Kemmis, S., McTaggart, R. y Nixon, R. (2013). The Action Research Planner. Doing Critical Participatory Action Research. Springer.

Latorre, A. (2005). La investigación-acción. Conocer y cambiar la práctica educativa. Grao.

Mancovsky, V. y Moreno, M. (2015). La formación para la investigación en el posgrado. Noveduc.

Mieg, HÁ. (2019). Introducción: Aprendizaje basado en la investigación - Evaluación inicial. En: Aprendizaje basado en la investigación - Investigación de pregrado. Springer, Cham.

McKernan, J. (1999). Investigación-acción y curriculum. Métodos y recursos para profesionales reflexivos. Morata.

Moriña, A. (2017). Investigación con historias de vida. Narcea.

Parra-Moreno, C. (2004). Apuntes sobre la investigación formativa. Educación y Educadores, (7), 57-77. https:/educacionyeducadores.unisabana.edu.co/index.php/eye/ article/view/549

Perkins, D. (2008). La escuela inteligente, del adiestramiento de la memoria a la educación de la mente ( $1^{\mathrm{a}}$. Ed. $4^{\circ}$ reimp.). Gedisa.

Schettini, P. y Cortazzo, I. (2015). Análisis de datos cualitativos en la investigación social. Procedimientos y herramientas para la interpretación de información cualitativa. Edulp.

Serulnikov, A. y Suárez R. (2010). Piaget para principiantes (1 ${ }^{\mathrm{a}}$. Ed. $3^{\circ}$ reimp.). Era Naciente.

Stenhouse, L. (2007). La investigación como base de la enseñanza ( $6^{\mathrm{a}}$. Ed. $1^{\circ}$ reimp.). Morata. 
Stone Wiske, M. (2008). La enseñanza para la comprensión: vinculación entre la investigación y la práctica $\left(1^{\mathrm{a}}\right.$. Ed. $3^{\circ}$ reimp.) Paidós.

Torres, A. (s.f.). Aprendizaje basado en la investigación. Técnicas didácticas. TEC de Monterrey. http://bit.ly/2woaZx5

Wiemer, M. (2019). Aprendizaje a traves de la investigación: aprendizaje independiente. Procesos de autoaprendizaje y habilidades de autoaprendizaje en el aprendizaje basado en la indagación. En: Mieg HA (eds) Aprendizaje basado en la investigación - Investigación de pregrado. Springer, Cham. 